\title{
ГРУППОВОЙ ПОДХОД В ДИНАМИКЕ МНОГОСПИНОВЫХ СИСТЕМ. III
}

В порядке приложения группового подхода $\left.{ }^{[}\right]$первыми исследовались преобразования движения, заданные над унимодулярной унитарной группой в виде произведения двух более простых преобразований $\left[{ }^{2}\right]$. Это привело к обобщению понятия резонанса как полного. Данная статья посвящена изучению частичных резонансов. Оказывается, что разные частичные резонансы связаны с динамическими подгруппами унимодулярной группы. Неприводимые подгруппы позволяют обобщить тиклинг-эффект, приводимые описывают обстановку, более близкую к опытам по коллапсу. Точная физическая реализация этих подгрупп требует возбуждения системы с помощью взаимодействий с определенными свойствами симметрии. Однако на основе резонанса возможна приближенная реализация менее специфическими взаимодействиями.

Результаты статьи применимы практически ко всем квантовым системам, обладающим конечным числом уровней, если только пренебрежение релаксацией и радиационным затуханием - основными условиями адиабатических процессов - оправдано.

\section{7. Селективное резонансное возбуждение}

7.1. Унимодулярная группа резонансного возбуж д ен и я. Исследуем $d$-уровневую систему с гамильтонианом $H_{0}$. Условимся нумеровать уровни $\omega_{m}^{(0)}(m=1,2, \ldots, d)$ оператора $H_{0}$ (уровни энергии) сверху вниз, т. е. $n>m$, если $\omega_{n}^{(0)}<\omega_{m}^{(0)}$.

Унимодулярная динамическая группа $\mathbf{u}_{d}$ называется группой резонансного возбуждения ( $d$-уровневой системы), если над $\mathbf{u}_{d}$ определены преобразования движения (6.27) со всевозможными $G, F \in \mathbf{u}_{d}^{0}$ (см. п. 6.2). Различные опыты, описываемые этой группой, отличаются выбором возбуждения $H_{1}(t)$ в гамильтониане $(6.14)$, т. е. выбором пары операторов $G, V$.

Полезно представить пространство $\mathbf{H}^{0}=\mathbf{u}_{d}^{0}$ всех эрмитовых операторов, имеющих нулевой след, в виде прямой суммы

$$
\mathbf{H}^{0}=\mathbf{H}_{A}^{0}+\mathbf{H}_{\perp} .
$$

$(d-1)$-мерное подпространство $\mathbf{H}_{A}^{0}$ содержит эрмитовые операторы (с нулевым следом), имеющие собственные векторы $a_{m}(m=1,2, \ldots, d) \in$ $\in \mathbf{C} . d(d-1)$-мерное подпространство $\mathbf{H}_{\perp}$ натянуто на эрмитовые базисные операторы $X_{m n}, Y_{m n}$ (см. п. 2.2) переходов $m \rightarrow n$ $(m, n=1,2, \ldots, d ; m<n)$. Согласно п. $6 H_{0}, G, \Delta \in \mathbf{H}_{A}^{0}$, но $H_{1}(t) \in \mathbf{H}_{\perp}$

Каждой паре операторов $\Delta, V$ соответствует оператор $T \in \mathbf{u}_{d}$ преоб- 
разования базисов $(6.30),(6.31)$. В частности, базис $A_{m m}$ пространства $\mathbf{H}_{A}$ переводится в базис $E_{m m}$ пространства $\mathbf{H}_{F}$, а $\mathbf{H}_{A}^{0}$ переходит в $(d-1)$ мерное подпространство $\mathbf{H}_{F}^{0} \subset \mathbf{H}_{F}$, содержащее оператор $F$ (6.25). Пространство $\mathbf{H}^{0}$ состоит из непрерывного множества подпространств типа $\mathbf{H}_{F}^{0}$, получаемых при разных парах $\Delta, V$. В частности, пределу полной расстройки соответствует $\mathbf{H}_{F}^{0}=\mathbf{H}_{A}^{0}$, а в точке полного резонанса $(6.45)-$ $V \in \mathbf{H}_{F}^{0} \subset \mathbf{H}_{\perp}$.

В пп. 6.1 и 6.2 движение $\varrho(t)$ представлялось в виде наложения двух движений - переносного (6.10), (6.41) и относительного (6.40). Состояния $\varrho \in \mathbf{H}_{A}$ не изменяются под влиянием переноса $\Re_{1}(t, 0)$. Состояния же $\varrho \in \mathbf{H}_{\perp}$ совершают вращения (5.19) с круговыми частотами $v_{m n}$ (6.36). Наложение относительного движения (6.40) не изменяет абсолютное движение $\varrho(t)$ лишь при начальных состояниях $\varrho(0) \in \mathbf{H}_{F}$. Перевод системы в такое состояние можно истолковать как обобщение метода «захвата спина» (spin-locking). В частности, в случае быстрого адиабатического прохождения, в приведенном здесь обобщенном смысле, движение начинается с состояния $\varrho(0) \in \mathbf{H}_{A}$ и заключается в медленной развертке всех $v_{m n}$ через точку полного резонанса (6.45). В любой момент времени

$$
\varrho(t)=\Re_{1}(t, 0) \varrho(0),
$$

а в спектре $Q$-наблюдаемой $(6.44)$ появляются лишь частоты $v_{m n}$.

В случае произвольного начального состояния $\varrho(0)$ движение $\varrho(t) \in$ $\in \mathbf{H}^{0}$ описывается формулами

$$
\begin{aligned}
& \varrho(t)=\varrho_{F}(t)+\sum_{j<k} \sum|\varrho(0)|_{j k}\left[\cos \left(\omega_{j k}^{(1)} t+\varphi_{j k}\right) X_{j k}^{(F)}(t)+\right. \\
& \left.+\sin \left(\omega_{j k}^{(1)} t+\varphi_{j k}\right) Y_{j k}^{(F)}(t)\right] \text {, } \\
& X_{j k}^{(F)}(t)=\Re_{1}(t, 0) X_{j k}^{(F)} \text {, } \\
& \varrho_{F}(t)=\Re_{1}(t, 0) \varrho_{F}(0), \\
& X_{j k}^{(F)}=T X_{j k} T^{-1} .
\end{aligned}
$$

Определения $Y_{j k}^{(F)}, \underset{j k}{Y_{j k}}(t)$ эквивалентны (7.6), (7.4); $|\mathrm{g}(0)|_{j k}-$ амплитуда проекции $\varrho(0)$ на плоскость $X_{j k}^{(F)}, \underset{j k}{Y_{j k}^{(F)}} ; \varrho_{F}(0)-$ компонента $\varrho(0)$ в пространстве $\mathbf{H}_{F}^{0}$.

Формула (7.5) аналогична формуле (7.2). В спектре $Q$-наблюдаемой компонента $Q_{F}(t)$ обеспечивает центральные частоты $v_{m n}$ субмультиплетов (см. ниже).

Второе слагаемое выражения (7.3) описывает вращения компонент $\varrho(t)$ на плоскостях $X_{j k}^{(F)}, \underset{j k}{Y_{j k}^{(F)}}$, сопровождаемые переносным движением (7.4) этих плоскостей. Проекция таких вращений на любую из плоскостей $X_{m n}, Y_{m n}$ приводит к картине сложного амплитудно-модулированного вращения. В спектре $Q$-наблюдаемой появляются субмультиплеты. Центральная частота $v_{m n}$ каждого субмультиплета сопровождается $d(d-1)$ боковыми частотами $v_{m n} \pm \omega_{j k}^{(1)}\left(\omega_{j R}^{(1)}>0\right)$, симметрично расположенными по обе ее стороны.

Более конкретное предсказание частот $\mathrm{Q}(t)$ и сигналов требует расчета частот $\omega_{j k}^{(1)}$ и базиса $E_{k k}, X_{j k}^{(F)}, Y_{j k}^{(F)}$ в зависимости от $\Delta, V$. В силу 
резонансного поведения системы существуют две частные области, описание которых в значительной мере исчерпывает всю проблему: 1) область достаточно слабого возмущения $V$ (резонансное приближение) и 2) область специально выбранных $H_{1}(t)$, обеспечивающих селективное возбуждение наборов переходов даже при сильном $V$.

7.2. Групп а селекти вного воз буждения. Это подгруппа $\mathbf{G} \subset \mathbf{u}_{d}$ группы резонансного возбуждения $\mathbf{u}_{d}$ с $H_{1}(t) \in \mathbf{H}_{V} \subset \mathbf{G}^{0}$, расположенными лишь в некотором подпространстве $\mathbf{H}_{V} \subset \mathbf{H}_{\perp}$.

В отличие от (7.1) динамическое кольцо $\mathbf{G}^{0}$ группы $\mathbf{G}$ можно представить в виде прямой суммы

$$
\mathbf{G}^{0}=\mathbf{H}_{A}^{0}+\mathbf{H}_{V},
$$

откуда

$$
\mathbf{H}_{\perp}=\mathbf{H}_{V}+\mathbf{H}_{K} \text {. }
$$

Помимо преобразований движения $D(t, 0)$, к группе $\mathbf{G}$ принадлежат также преобразования базиса $T$.

Подгруппа симметрии $\mathbf{S} \subset \mathbf{G}$ группы $\mathbf{G}$ селективного возбуждения состоит из элементов $\boldsymbol{S} \in \mathbf{S}$, оставляющих неизменными элементы $\mathbf{G}$ и $\mathbf{G}^{0}$. Например,

$$
S H(t) S^{-l}=H(t) \text {. }
$$

Инфинитизимальное кольцо $\mathbf{S}^{0} \subset \mathbf{G}^{0}$ группы симметрии $\mathbf{S}$ состоит из эрмитовых операторов, коммутирующих со всеми элементами $\mathbf{G}^{0}$ и имеющих нулевой след. $\mathbf{S}^{0}$ образует подпространство в $\mathbf{H}_{A}^{0}$. Поэтому $\operatorname{dim} \mathbf{S}^{0}<(d-1)$.

Если $\mathbf{S}^{0}$ существует, то $\mathbf{H}_{A}^{0}$ распадается на прямую сумму

$$
\mathbf{H}_{A}^{0}=\mathbf{S}^{0}+\mathbf{H}_{L}^{0} .
$$

В этом случае подпространство

$$
\mathbf{G}_{L}^{0}=\mathbf{H}_{L}^{0}+\mathbf{H}_{V} \subset \mathbf{G}^{0}
$$

само образует динамическое кольцо, соответствующее некоторой подгруппе $\mathbf{G}_{L} \subset \mathbf{G}$. Поэтому нахождение возможных $\mathbf{S}^{0} \subset \mathbf{H}_{A}^{0}$, коммутирующих с некоторым кольцом $\mathbf{G}_{L}^{0}$, есть один из способов выделения групп селективного возбуждения.

Для доказательства того, что ортогональное дополнение $\mathbf{G}_{L}^{0}$ к $\mathbf{S}^{0} \subset \mathbf{G}^{0}$ образует кольцо, достаточно показать, что -i $\left[G_{1}, G_{2}\right]$ с любыми $G_{1}, G_{2} \in \mathbf{G}_{L}^{0}$ ортогонален с любым $S^{0} \in \mathbf{S}^{0}$. Так как след трех эрмитовых операторов $G_{1}, G_{2}, S^{0}$ не изменяется при циклической перестановке

$$
\operatorname{tr}\left(G_{1} G_{2} S^{0}\right)=\operatorname{tr}\left(S^{0} G_{1} G_{2}\right),
$$

то, в силу коммутирования элементов $\mathbf{S}^{0}$ и $\mathbf{G}_{L}^{0}$, получаем

$$
\left(-i\left[G_{1}, G_{2}\right], S^{0}\right)=\left(-i\left[S^{0}, G_{1}\right], G_{2}\right)=0 .
$$

Итак, $\mathbf{G}_{L}^{0}$ дополняется до $\mathbf{G}^{0}$

$$
\mathbf{G}^{0}=\mathbf{S}^{0}+\mathbf{G}_{L}^{0},
$$


а пространство $\mathbf{H}^{0}$ разлагается на прямую сумму подпространств

$$
\mathbf{H}^{0}=\mathbf{S}^{0}+\mathbf{G}_{L}^{0}+\mathbf{H}_{K},
$$

инвариантных относительно супероператорного представления группы G. Подпространства типа $\mathbf{H}_{F}^{0}$ имеют $\operatorname{dim} \mathbf{H}_{F}^{0}=\operatorname{dim} \mathbf{H}_{L}^{0}$ и заполняют подпространство $\mathbf{G}_{L}^{0} \subset \mathbf{H}^{0}$.

Разложению (7.14) соответствует разложение всех операторов $\mathbf{G}^{0}$ на компоненты, лежащие в $\mathbf{S}^{0}$ (индекс $S$ ) и в $\mathbf{G}_{L}^{0}$ (индекс $L$ ) соответственно:

$$
\begin{aligned}
H_{0} & =H_{S}+H_{L}, \\
G & =G_{S}+G_{L}=H_{S}+G_{L}, \\
\Delta & =\Delta_{L}=H_{L}-G_{L}, \\
F & =F_{L}=\Delta_{L}+V .
\end{aligned}
$$

Возбуждение $H_{1}(t)$ не зависит от выбора $G_{S}$ и определяется лишь парой $G_{L}, V$. Поэтому в $(7.17)-(7.19)$ принято $G_{S}=H_{S}$. Выражение же (6.27) следует заменить на

$$
D(t, 0)=\exp \left(-i t H_{S}\right) \exp \left(-i t G_{L}\right) \exp \left(-i t F_{L}\right) .
$$

Разложению (7.15) соответствует разложение $\varrho(t)$ на компоненты

$$
\mathrm{Q}(t)=1 / 4 E+\varrho_{S}(0)+\varrho_{L}(t)+\varrho_{K}(t) .
$$

Компонента os $\in \mathbf{S}^{0}$ вообще не изменяется. Временное изменение компоненты $\varrho_{L}(t) \in \mathbf{G}_{L}^{0}$ описывается супероператорным представлением группы резонансного возбуждения $\mathbf{G}_{L} \subset \mathbf{u}_{d}$, имеющей

$$
H_{L}(t)=H_{L}+H_{1}(t) \in G_{L}^{0}
$$

в качестве гамильтониана. Изменение же $\varrho_{K}(t) \in H_{K}$ определяется полным гамильтонианом $H(t)=H_{\mathrm{S}}+H_{L}(t)$.

Р-наблюдаемые (см. п. 5.4) типа $\mathrm{P} \in \mathbf{S}^{0}$ являются интегралами движения - их средние значения (5.37) постоянны во времени. Число боковых частот в субмультиплетах сокращается в соответствии с симметрией группы $\mathbf{G}$ (см. ниже).

Захват спина (см. п. 7.1) возможен лишь в пределах подпространства $\mathbf{G}_{L}^{0}$. В частности, при быстром адиабатическом прохождении $\varrho_{K}(t)=0$. Лишь в случае $\varrho_{K}(0) \neq 0$ наблюдаются сигналы, происходящие от $\varrho_{K}(t)$. В этих сигналах отражено влияние возбуждения $H_{1}(t)$.

В общем случае точка полного резонанса (6.45) недостижима (в группе (G). Возможен лишь частичный резонанс *

$$
G_{L}=H_{L}
$$

Собственные векторы $e_{m}$ любого оператора $F_{L}$ группы селективного возбуждения $\mathbf{G}$ (имеющей подгруппу симметрии $\mathbf{S}$ ) должны одновре-

* В изыскании точки полного резонанса (6.45) можно увидеть наиболее прямой спектроскопический метод измерения $H_{0}$. Нахождение точки частнчного резонанса (7.23) позволяет измерять часть $H_{L}$ гамильтониана $H_{0}$. Свойства симметрии взаимодействий $H_{L}, H_{1}(t)$ определяют возможность раздельного измерения $H_{L}$ этим способом. 
менно быть собственными векторами любого $S^{0} \in \mathbf{S}^{0}$. Это возможно лишь в том случае, когда $S^{0}$ имеет одно или несколько вырожденных собственных значений.

Пространство состояний $\mathbf{C}$ распадается на прямую сумму подпространств ${ }^{(q)} \mathbf{C}$

$$
\mathbf{C}=\sum_{q=1}^{l}(q) \mathbf{C},
$$

инвариантных относительно операторов $\mathbf{G}$ и $\mathbf{G}^{0}$. В частности, операторы $T \in \mathbf{G}$ переводят часть базиса $a_{m}, a_{n} \ldots \in(q) \mathbf{C}(q=1,2, \ldots, l)$ в часть базиса $e_{m}, e_{n}, \ldots \in(q) \mathbf{C}$. Любой $S^{0} \in \mathbf{S}^{0}$ имеет в качестве подпространства собственных векторов ${ }^{(q)} \mathbf{C}$, принадлежащие собственному значению с кратностью вырождения $d_{q}=\operatorname{dim}(q)$ C. Матрицы операторов $\mathbf{G}$ и $\mathbf{G}^{0}$ (на любом базисе типа $e_{m}$ ) либо квазидиагональны, либо приводимы к таковым путем перестановки рядов и столбцов. Поэтому операторы $D(t, 0) \in \mathbf{G}$ и $H(t) \in \mathbf{G}^{0}$ разлагаются на прямые суммы (3.4) и (3.5) соответственно. В этом смысле группа $\mathbf{G}$ и кольцо $\mathbf{G}^{0}$ - приводимые.

Изучение приводимых групп в значительной мере сводится к изучению самых простых групп селективного возбуждения - неприводимых.

7.3. Неприводимы е группы селективного возбуж дения и ади абатический тиклинг. Выделяем в прямой

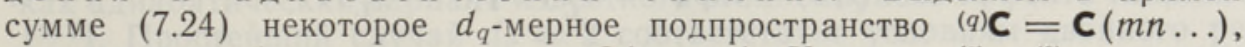
натянутое на базис $a_{m}, a_{n}, \ldots \in \mathbf{C}(m n \ldots)$. Уровни $\omega_{m}^{(0)}, \omega_{n}^{(0)} \ldots$ и все переходы $m \rightarrow n \ldots$ между ними будем называть возбуждаемыми. Два перехода $m \rightarrow k, k \rightarrow n$, имеющие общий уровень $k$, будем называть связанными и в противном случае - несвязанными. Переход $m \rightarrow n$ является замыкающим относительно связанных переходов $m \rightarrow k, k \rightarrow n$. Bсе переходы между уровнями подпространства $(q) \mathbf{C}$ образуют систему взаимосвязанных возбуждаемых переходов (обозначения номера $q$ и перечня $m n \ldots$ номеров возбуждаемых уровней эквивалентны). Графически этой системе соответствует схема уровней энергии, где возбуждаемые переходы (граф возбуждаемых переходов) обозначаются стрелками.

Группа селективного возбуждения $\mathbf{G}_{L}(m n \ldots)$ считается неприводимой, если над ней определены преобразования движения $D^{(q)}(t, 0)$, действующие как единичные операторы в подпространстве, ортогонально дополняющем ${ }^{(q)} \mathbf{C}$ (не смешивать с аналогичным обозначением в п. 3.3). Квазидиагональная матрица $D^{(q)}(t, 0)$ (на базисе $a_{m}$ ) имеет ненулевые недиагональные элементы лишь в блоке, соответствующем ${ }^{(q)} \mathbf{C}$.

Динамическое кольцо $\mathbf{G}_{L}^{(q)}=\mathbf{G}_{L}^{0}(m n \ldots)$ неприводимой группы $\mathbf{G}_{L}(m n \ldots)$ содержит гамильтонианы вида

$$
H_{L}^{(q)}(t)=H_{L}^{(q)}+H^{(q)}(t),
$$

действующие в ортогональном дополнении к ${ }^{(q)} \mathbf{C}$ как нулевые операторы. В данном случае формула (7.11) принимает вид

$$
\mathbf{G}_{L}^{(q)}=\mathbf{H}_{L}^{(q)}+\mathbf{H}_{V}^{(q)} .
$$

Имеем: $\quad H_{L}^{(q)} \in \mathbf{H}_{I_{L}}^{(q)} \subset \mathbf{H}_{A}^{0}$, но $H^{(q)}(t) \in \mathbf{H}_{V}^{(q)} \subset \mathbf{H}_{\perp}: \quad$ Ясно, что

$$
\operatorname{dim} \mathbf{H}_{L}^{(q)}=d_{q}-1,
$$




$$
\begin{aligned}
\operatorname{dim} \mathbf{H}_{V}^{(q)} & =d_{q}\left(d_{q}-1\right), \\
\operatorname{dim} \mathbf{G}_{L}^{(q)} & =d_{q}^{2}-1 .
\end{aligned}
$$

Подпространство $\mathbf{H}_{V}^{(q)}$ натянуто на базисные операторы $X_{m n}, Y_{m n}$ возбуждаемых переходов $m \rightarrow n$. Базисом подпространства может служить набор линейно независимых операторов $Z_{m n}$ (см. п. 2.2) возбуждаемых переходов $m \rightarrow n, \ldots$. Операторы $Z_{m n}, Z_{j k}$ несвязанных переходов ортогональны. Операторы же $Z_{m k}, Z_{k n}$ связанных переходов не ортогональны и, кроме того, не коллинеарны (угол между $Z_{m k}, Z_{k n}$, равный $120^{\circ}$, делится пополам вектором $Z_{m n}$ ). Оператор $Z_{m n}$ перехода $m \rightarrow n$, замыкающего переходы $m \rightarrow k, k \rightarrow n$, задан соотношением

$$
Z_{m n}=Z_{m k}+Z_{k n} \text {. }
$$

Поэтому, располагая $Z_{m k}, Z_{k n}$ в порядке увеличения индексов $m<k<n<\ldots$, получим (неортогональный) базис подпространства $\mathbf{H}_{L}^{(q)} \subset \mathbf{H}_{A}^{0}$.

Преобразования движения (6.27) группы $\mathbf{G}_{L}(m n \ldots)$ следует записать в виде

$$
D^{(q)}(t, 0)=\exp \left(-i t G_{L}^{(q)}\right) \exp \left(-i t F_{L}^{(q)}\right),
$$

где $G_{L}^{(q)} \in \mathbf{H}_{L}^{(q)}, \quad$ но

$$
F_{L}^{(q)}=\Delta_{L}^{(q)}+V^{(q)} \in \mathbf{G}_{L}^{(q)} .
$$

Оператор $T \in \mathbf{G}_{L}(m n \ldots)$ изменяет базис $a_{m}$ лишь в пределах подпространства ${ }^{(q)} \mathbf{C}$. Спектр собственных значений операторов кольца $\mathbf{G}_{L}^{(q)}$ состоит из вырожденного нулевого уровня и из $d_{q}$ невырожденных уровней, расположенных по обе стороны нулевого. Имеется $1 / 2 d_{q}\left(d_{q}-1\right)$ ненулевых частот $\omega_{j h}^{(1)}\left(a_{j}, a_{k} \in{ }^{q} \mathbf{C}\right)$. Оператор $H_{1}^{(q)}(t)$ задается суммой (6.21), содержащей только возбужденные переходы.

Группа $\mathbf{G}_{L}(m n \ldots)$ применима лишь в случае $H_{0}=H_{0}^{(q)} \in \mathbf{H}_{L}^{(q)} \subset \mathbf{H}_{A}$. Она может быть расширена до группы селективного возбуждения системы взаимосвязанных переходов (группы тиклинга $\mathbf{G}(m n \ldots))$ путем дополнения $\mathbf{H}_{L}^{(q)}$ до $\mathbf{H}_{A}^{0}$ прибавлением $\mathbf{S}^{0}$. В данном случае любой $S^{0} \in \mathbf{S}^{0}$ имеет $d_{q}$-кратно вырожденный уровень, соответствующий собственным векторам в (q) $\mathbf{C}$. Остальные уровни оператора $\mathbf{S}^{0}$ ограничены требованием $\operatorname{tr} S^{0}=0$.

Динамическое кольцо $\mathbf{G}^{0}(m n \ldots)$ группы тиклинга: $\mathbf{G}^{0}(m n \ldots)=$ $=\mathbf{S}^{0}+\mathbf{G}_{L}^{0}(m n \therefore)$. Оно содержит операторы $H_{0}=H_{S}+H_{L}^{(q)}, G=G_{L}^{(q)}$, $H(t)=H^{(q)}(t), F=F_{L}^{(q)}$. Согласно (7.20), выражение (7.29) в случае $\mathbf{G}(m n \ldots)$ дополняется членом, включающим $H_{S}$.

В случае группы тиклинга разложение (7.15) можно уточнить следующим образом:

$$
\mathbf{H}^{0}=\mathbf{S}^{0}+\mathbf{G}_{L}^{0}(m n \ldots)+\mathbf{H}_{K 1}+\mathbf{H}_{K 2} .
$$

Движение компоненты $\varrho_{L}(t) \in \mathbf{G}_{L}^{0}(m n \ldots)($ см. 7.21$)$ совпадает с движением $d_{q}$-уровневой системы с гамнльтонианом (7.25). В частности, субмультиплеты имеют центральную частоту $v_{m n}\left(a_{m}, a_{n} \in{ }^{(q)} \mathbf{C}\right)$ и 
$d_{q}\left(d_{q}-1\right)$ боковых частот $\quad v_{m n} \pm \omega_{j k}^{(l)} \quad\left(a_{j}, a_{k} \in(q) \mathbf{C}\right)$. Учет $H_{S}$ сдвигает частоты $v_{m}$ на «правильное» положение, соответствующее гамильтониану $H_{0}$. Условие частичного резонанса $(7.23)$ имеет вид

$$
G_{L}^{(q)}=H_{L}^{(q)},
$$

т. е. условие резонанса $v_{m n}=\omega_{m n}^{(0)}$ выполнимо одновременно лишь для возбуждаемых переходов $m \rightarrow n, \ldots$

Подпространство $\mathbf{H}_{K 1}$ натянуто на базисные операторы $X_{m k}, Y_{m k}$ переходов $m \rightarrow k, \ldots$, связанных с возбуждаемыми переходами. Такие переходы имеют лишь один возбуждаемый уровень - либо $a_{m} \in{ }^{(q)} \mathbf{C}$, либо $a_{k} \in{ }^{(q)} \mathbf{C}$. Подпространство $\mathbf{H}_{K 2}$ опирается на базисные операторы переходов между невозбуждаемыми уровнями. Операторы подпространств $\mathbf{G}_{L}^{0}(m n \ldots)$ и $\mathbf{H}_{K 2}$ коммутируют не с операторами подпространства $\mathbf{H}_{K 1}$, а между собой.

Преобразование (7.6) не затрагивает базиса подпространства $\mathbf{H}_{K 2}$. Если компонента $\mathrm{Q}(0)$ в $\mathbf{H}_{K 2}$ существует, то движение в нем совершается частотами свободного движения $\omega_{m n}^{(0)}\left(a_{m}, a_{n}-\right.$ невозбуждаемые).

Характерные особенности динамики группы тиклинга проявляются в движении компоненты $\mathrm{QK}_{K}(t)$ в подпространстве $\mathbf{H}_{K 1}$-переходов, связанных с возбуждаемыми переходами. Эту динамику можно рассматривать как адиабатический вариант известного стационарного эффекта тиклинга $\left[{ }^{3,4}\right]$. Простейший случай $d_{q}=2$ соответствует условиям двойного резонанса [ $\left.{ }^{4}\right]$, случай $d_{q}=3$ - условиям тройного резонанса $\left[{ }^{5,6}\right]$. (При адиабатическом варианте т. н. измерительное радиочастотное поле не нужно. Но на связанном переходе должно быть предварительно возбуждено свободное движение.)

Воспользуемся выражением

$$
D(t, 0)=\exp \left(-i t\left(H_{0}-\Delta_{L}\right)\right) \exp \left(-i t F_{L}\right),
$$

эквивалентным формуле (7.20). В случае группы тиклинга: $\Delta_{L}=\Delta_{L}^{(q)}$; $F_{L}=F_{L}^{(q)} . \quad$ Ортогональное супероператорное представление преобразования (7.33) приводит к формуле типа (7.3) с особенностями, присущими группе тиклинга. Обсудим эти особенности для компоненты $l$ в $\mathbf{H}_{K 1}$.

Пусть индексы $m \in q$ и $l \in p$ относятся к возбуждаемому и, соответственно, невозбуждаемому уровням энергии. Рассмотрим плоскость перехода $m \rightarrow l$. По (7.33) на этой плоскости круговая частота переносного движения равна ${ }^{(0)}{ }_{m l}-\Delta v_{m l}=\omega_{m l}^{(0)}-\Delta v_{m} \quad$ (поскольку $\left(d-d_{q}\right)$. кратно вырожденный уровень $l \in p$ оператора $\Delta_{L}^{(q)}$ нулевой). Это частота переноса проекции всех (7.4) на плоскости $X_{m l}, Y_{m l}$. Но так как $\mathbf{H}_{F}^{0} \subset \mathbf{G}_{L}^{(q)}, \quad$ то сама центральная частота субмультиплета (см. п. 7.1) в сигнале все же отсутствует. В сумме (7.3) - двойная сумма: $j \in q$, $k \in p$. Но $\omega_{j k}^{(1)}=\omega_{j}^{(1)}$. Поэтому в спектре $Q$-наблюдаемой переходу $m \rightarrow l$ соответствует субмультиплет из $d_{q}$ боковых частот

$$
\left(\omega_{m l}^{(0)}-\Delta v_{m}+\omega_{j}^{(l)}\right) ; \quad j \in q .
$$

В формуле (6.30) $e_{k}=a_{k}$ при $k \in p$. Поэтому выражение (6.31) упрощается:

$$
E_{j k}=\sum_{m \in q} T_{m j} A_{m k} ; \quad j \in q, \quad k \in p .
$$


Обозначая

$$
T_{m j}=\left|T_{m j}\right| \exp \left(-i \vartheta_{m j}\right),
$$

запишем преобразования (7.6) в $\mathbf{H}_{K 1}$ в виде

$$
\begin{aligned}
& X_{j k}^{(F)}=\sum_{m \in q}\left|T_{m j}\right|\left(\cos \vartheta_{m j} X_{m k}+\sin \vartheta_{m j} Y_{m k}\right), \\
& Y_{j k}^{(F)}=\sum_{m \in q}\left|T_{m j}\right|\left(-\sin \vartheta_{m j} X_{m k}+\cos \vartheta_{m j} Y_{m k}\right) .
\end{aligned}
$$

В (7.37) и в дальнейшем предполагается, что $m<k$. В противном случае знак перед $Y_{m k}$ следует поменять.

По (7.37) пространство $\boldsymbol{H}_{K 1}$ распадается на прямую сумму $\left(d-d_{q}\right)$ подпространств, инвариантных относительно ортогонального супероператорного представления группы тиклинга. Каждому $k \in p$ соответствует инвариантное подпространство, состоящее из $d_{q}$-плоскостей $X_{m k}$, $Y_{m k}$ переходов $m \rightarrow k, m \in q$.

Подстановка (7.37) в (7.4) и (7.3) позволяет вычислить проекцию $\varrho(t)_{m l}$ оператора $\varrho(t)$ на плоскость перехода $m \rightarrow l$

$$
\begin{gathered}
\varrho(t)_{m l}=\sum_{j \in q}\left|T_{m j}\right||\varrho(0)|_{j l}\left[\cos \left(\omega_{j}^{(l)} t+\varphi_{j l}+\vartheta_{m j}\right) X_{m l}(t)+\right. \\
\left.\quad+\sin \left(\omega_{j}^{(l)} t-1-\varphi_{j l}+\vartheta_{m j}\right) Y_{m l}(t)\right] .
\end{gathered}
$$

Итак, расчет движения (7.38) (и соответствующих сигналов) предполагает: 1) нахождение собственных значений $\Delta v_{m}, \omega_{j}^{(1)}$ операторов $\Delta_{L}^{(q)}$ и $\underset{L}{F(q)} \quad$ соответственно и 2$)$ вывод оператора $T$ в виде матрицы на базисе $a_{m}$.

Согласно $(7.38)$, проекция $\varrho(t)_{m l}$ совершает на плоскости $X_{m l}, Y_{m l}$ вращение с угловой частотой $\left(\omega_{m l}^{(0)}-\Delta v_{m}\right)$ и амплитудой, осциллирующей с набором частот $\omega_{j}^{(l)}$. Это приводит к наблюдаемому субмультиплету (7.34). В наиболее простом случае $d_{q}=2$ (лишь один возбуждаемый переход) получаем дублет, расщепление которого задается формулой, аналогично той, которая получена в $\left[{ }^{4}\right]$ для стационарного тиклинга.

7.4. Приводимы е группы селективного возбужде ния. Располагая набором $l$ неприводимых групп $\mathbf{G}(m n \ldots)$, можно сконструировать приводимую группу селективного (резонансного) возбуждения (ср. с п. 3.2). В качестве элементов такой группы и ее динамического кольца имеем соответственно

$$
\begin{aligned}
D(t, 0) & =\exp \left(-i t H_{S}\right) D^{(1)}(t, 0) D^{(2)}(t, 0) \ldots, \\
H(t) & =H_{S}+H_{L}^{(1)}(t)+H_{L}^{(2)}(t)+\ldots .
\end{aligned}
$$

Динамическое кольцо состоит из прямой суммы взаимно коммутирующих неприводимых подколец

$$
\mathbf{G}^{0}=\mathbf{S}^{0}+\sum_{q=1}^{l} \mathbf{G}_{L}^{(q)},
$$

каждое из которых имеет структуру типа (7.26). Поэтому 


$$
\begin{gathered}
\mathbf{H}_{A}^{0}=\mathbf{S}^{0}+\sum_{q=1}^{l} \mathbf{H}_{L}^{(q)}, \\
\mathbf{H}_{V}=\sum_{q=1}^{l} \mathbf{H}_{V}^{(q)} .
\end{gathered}
$$

В данном случае подкольцо симметрии $\boldsymbol{S}^{0}$ содержит лишь общую часть всех $\mathbf{S}^{0}$ групп тиклинга, составляющих данную приводимую группу. Любой $S^{0} \in \mathbf{S}^{0}$ имеет вырожденные уровни, соответствующие отдельным слагаемым в прямой сумме (7.24). Матрицы операторов (7.39), (7.40) (на базисе $a_{m}$ ) имеют квазидиагональный вид (3.4) и (3.5). Разложения (7.16) - (7.19) уточняются:

$$
\begin{aligned}
& H_{0}=H_{S}+\sum_{q=1}^{l} H_{L}^{(q)}, \\
& G=G_{L}=\sum_{q=1}^{l} G_{L}^{(q)}, \\
& \Delta=\Delta_{L}=\sum_{q=1}^{l} \Delta_{L}^{(q)}, \\
& F=F_{L}=\sum_{q=1}^{l} F_{L}^{(q)} .
\end{aligned}
$$

Взамен одного условия резонанса (7.23) можно сформулировать $l$ условий частичного резонанса типа (7.32). Лишь при выполнении всех этих условий (точном или приближенном) относительное движение проявляется во всех подпространствах $\mathbf{G}_{L}^{(q)}$. Если же некоторая $\left|\Delta_{L}^{(q)}\right|$ слишком велика, то в хорошем приближении движение описывается подгруппой группы G. Переводя в $(7.42) \underset{L}{H} H_{L}^{(q)}$ в состав $\mathbf{S}^{0}$, а в (7.43) $H_{V}^{(q)}$ в состав $\mathbf{H}_{K}$, получим динамическое кольцо новой подгруппы. Итак, приводимая группа $\mathbf{G}$ содержит иерархию новых приводимых групп в качестве подгрупп $\mathbf{G}$.

Ортогональное супероператорное представление приводимой группы состоит из неприводимых множителей

$$
\Re(t, 0)=\Re^{(0)}(t, 0) \Re^{(1)}(t, 0) \Re^{(2)}(t, 0) \ldots
$$

В выражении $(7.48) \mathfrak{R}^{(0)}(t, 0)$ соответствует первому множителю в $(7.39), \Re^{(q)}(t, 0)$ при $q=1,2, \ldots-$ множителю $D^{(q)}(t, 0)$.

Согласно п. 4.3, разложение $\mathbf{H}^{0}(7.15)$ на инвариантные подпространства представления (7.48) уточняется следующим образом:

$$
\mathbf{H}^{0}=\mathbf{S}^{0}+\sum_{q=1}^{l} \mathbf{G}_{L}^{(q)}+\sum_{q<p} \sum_{<} \mathbf{H}^{(q p)} .
$$

Подпространство $\mathbf{H}^{(q p)} \subset \mathbf{H}_{K}$ натянуто на базисные операторы $X_{m l}, Y_{m l}$ переходов типа $m \rightarrow l, m \in q, l \in p$. Эти переходы связывают возбуж-

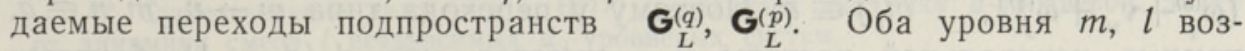
буждаемы. Но сам переход - невозбуждаем.

Баланс размерностей дает

$$
\begin{aligned}
\operatorname{dim} \mathbf{S}^{0} & =l-1, \\
\operatorname{dim} \mathbf{H}^{(q p)} & =2 d_{q} d_{p},
\end{aligned}
$$




$$
\operatorname{dim} \mathbf{H}_{K}=d^{2}-\sum_{q=1}^{l} d_{q}^{2}
$$

Граф возбуждаемых переходов приводимой группы $\mathbf{G}$ состоит из $l$ подсистем взаимосвязанных возбуждаемых переходов. Между отдельными подсистемами нет связывающих возбуждаемых переходов. В обозначенной через $\mathbf{G}=\mathbf{G}(m n, j k l, \ldots)$ приводимой группе запятыми отделяем номера возбуждаемых уровней, принадлежащих к разным подсистемам. Например: $\mathbf{G}^{0}(12,34)$ четырехуровневой системы содержит, помимо одномерного $\mathbf{S}^{0}$, 3-мерные подкольца $\mathbf{G}_{L}^{0}(12)$ и $\mathbf{G}_{L}^{0}$

(34). Возбуждены переходы $1 \rightarrow 2,3 \rightarrow 4 . \mathbf{H}_{K}=\mathbf{H}^{(12)}$ включает переходы $1 \rightarrow 3,2 \rightarrow 4,1 \rightarrow 4,2 \rightarrow 3$.

Поясним вопрос о собственных значениях операторов выражений $(7.44)-(7.47)$ и соответствующих им супероператоров.

При $m \in q$ величина $v_{m}$ есть собственное значение как оператора $G$, так и оператора $G_{L}^{(q)}$. Аналогично, $\Delta v_{m}$ есть (ненулевое) собственное значение операторов $\Delta, \Delta_{L}^{(q)}$, а $\omega_{j}^{(l)}-$ операторов $F, F_{L}^{(q)}(j \in q)$. Если собственные значения операторов $H_{S}$ и $H_{L}^{(q)}$, относящихся к уровню $m \in q$, обозначить временно через $\omega_{q}^{(0)}$ и $\omega_{m}^{(q)}$ соответственно, то собственное значение $\underset{m}{\omega_{m}^{(0)}}$ оператора $H_{0}$ будет равняться

$$
\omega_{m}^{(0)}=\omega_{q}^{(0)}+\omega_{m}^{(p)}
$$

Поэтому при $m, n \in q$ имеем

$$
\omega_{m n}^{(0)}=\omega_{m n}^{(q)}
$$

а при $m \in q, l \in p-$

$$
\omega_{m l}^{(0)}=\omega_{q p}^{(0)}+\left(\omega_{m}^{(q)}-\omega_{l}^{(p)}\right) .
$$

В силу (7.20) круговая частота переносного движения (частота центральной линии субмультиплета) для перехода $m \rightarrow n, m, n \in q$, равняется $v_{m n}$, а для перехода $m \rightarrow l, m \in q, l \in p$ соответственно $\left(\omega_{q p}^{(0)}+v_{m l}\right)$.

Возникновение субмультиплетов можно наглядно проследить на графе возбужденных уровней (и переходов) оператора $H_{\mathrm{S}}+G_{L}=$ $=H_{0}-\Delta_{L}$, дополненного системой субуровней. На этом графе любой уровень $\omega_{q}^{(0)}+v_{m}=\omega_{m}^{(0)}-\Delta v_{m}, \quad m \in q$, расщепляется на $d_{q}$ субуровней $\left(\omega_{q}^{(0)}+v_{m}+\omega_{j}^{(1)}\right), \quad m, j \in q$. Поэтому у перехода типа $m \rightarrow n, m, n \in q$, помимо центральной частоты $v_{m n}$ имеется еще $d_{q}\left(d_{q}-1\right)$ боковых частот

$$
v_{m n} \pm \omega_{j k}^{(1)}=\omega_{m n}^{(0)}-\Delta v_{m n} \pm \omega_{j k}^{(1)}
$$


где $m, n, j, k \in q$. В случае же перехода подпространства $\mathbf{H}^{(q p)} m \rightarrow l$, $m \in q, l \in p$, центральной линии нет, а $d_{q} d_{p}$ боковых частот субмультиплета вычисляются по

$$
\omega_{q p}^{(0)}+v_{m l}+\omega_{j k}^{(1)}=\omega_{m l}^{(0)}-\Delta v_{m l}+\omega_{j k}^{(1)},
$$

где $m, j \in q$, но $l, k \in p$.

Базис подпространства $\mathbf{H}_{A}^{0}$, приспособленный к данной приводимой группе, состоит, во-первых, из базисных операторов $B_{k}(k=1,2, \ldots, l)$ подпространства $\mathbf{S}^{0}$ и, во-вторых, из линейно независимых операторов $Z_{m n}$ (см. п. 7.3), сгруппированных по подпространствам $\mathbf{H}_{L}^{(q)}$. Операторы $X_{m n}, Y_{m n}$ по-прежнему удобно использовать в качестве базиса в $\mathbf{H}_{\perp}$. Их тоже следует сгруппировать по подпространствам $\mathbf{H}_{V}^{(q)}$ и $\mathbf{H}^{(q p)}$. Аналогично (7.48), преобразование (7.6) этого базиса состоит из неприводимых множителей.

Движение компоненты $\varrho(t)$ в подпространстве $\underset{L}{\mathbf{G}_{(}^{(q)}}$ описывается супероператором $\Re^{(q)}(t, 0)$. Это - динамика унимодулярной группы резонансного возбуждения (см. п. 7.1) с размерностью $d_{q}-1$.

Движение компоненты $\varrho(t)$ в подпространстве $\mathbf{H}^{(q p)}$ описывается произведением $\Re^{(0)}(t, 0) \Re^{(q)}(t, 0) \Re^{(p)}(t, 0)$. ** В частности, переносное движение (7.4)

$$
X_{j k}^{(F)}(t)=\Re_{1}^{(0)}(t, 0) \Re_{1}^{(q)}(t, 0) \Re_{1}^{(p)}(t, 0) X_{j k}^{(F)}
$$

базиса (7.6) с $j \in q, k \in p$, происходит с круговой частотой

$$
\omega_{q p}^{(0)}+\left(v_{m}-v_{l}\right)=\omega_{m l}^{(0)}-\Delta v_{m l},
$$

где

$$
\Delta v_{m l}=\Delta v_{m}-\Delta v_{l} .
$$

Действующее на систему возбуждение $H_{1}(t)$ не имеет в сумме $(6.21)$ частоты $v_{m l}$. Поэтому расстройка (7.58) является функцией расстроек частот возбуждения на возбуждаемых переходах подсистем $q$ и $p$. Эта зависимость получается на основании разложения $\Delta_{L}^{(q)}$ и $\Delta_{L}^{(p)}$ по базисным операторам типа $Z_{m n}$.

Установление базиса (7.6) относительного движения в $\mathbf{H}^{(q p)}$ сводится к двум последовательным преобразованиям типа (7.37). В результате $X_{j k}^{(F)} \in \mathbf{H}^{(q p)},-j \in q, k \ni p$, приобретает компоненты на всех базисных операторах переходов, совершающихся между уровнями $q$ и $p$ подсистем и сходящихся на уровни $j$ и $k$. Прилагая затем формулу (7.3), получаем детальную картину всех движений и сигналов.

Итак, описание приводимой группы селективного возбуждения сводится к описанию набора неприводимых групп. В частности, резонансное поведение системы определяется набором частичных резонансов неприводимых групп.

** Существование движения в $\mathbf{H}^{(q p)}$ и отличает динамику $d$-уровневой системы от динамики смеси $l$ независимых $d_{q}$-уровневых систем.

\section{Л ИТ Е РАТ У РА}

1. Синив в э В., Изв. АН ЭССР, Физ. Матем., 24, 35 (1975).

2. Си ни ве э В., Изв. АН ЭССР, Физ. Матем., 25, 146 (1976).

3. Autler, S. H., Townes, C. H., Phys, Rev., 100, 703 (1955), 
4. Freem a n, R., Anders o n, W. A., J. Chem. Phys., 37, 2053 (1962).

5. С ин и в е э В., Изв. АН ЭССР, Физ. Матем., 16, 444 (1967).

6. B y st r ov, V. F., J. Molec. Spectr., 28, 81 (1968).

\section{Ннститут кибернетики}

Академии наук Эстонской ССР
Поступила в редакцию 2/VI 1976

\section{SINIVEE}

ROHMADE TEOORIA RAKENDAMISEST MITMESPINNISUSTEEMIDE DUNAAMIKA UURIMISEKS. III

Artiklis käsitletakse mitmespinnisüsteemide selektiivse ergastamise seost dünaamiliste rühmadega.

V. SINIVEE

\section{GROUP APPROACH IN DYNAMICS OF MANY SPIN SYSTEMS. III}

The relations between dynamical groups and selective resonance excitation of manylevel quantum systems are studied in detail. 